
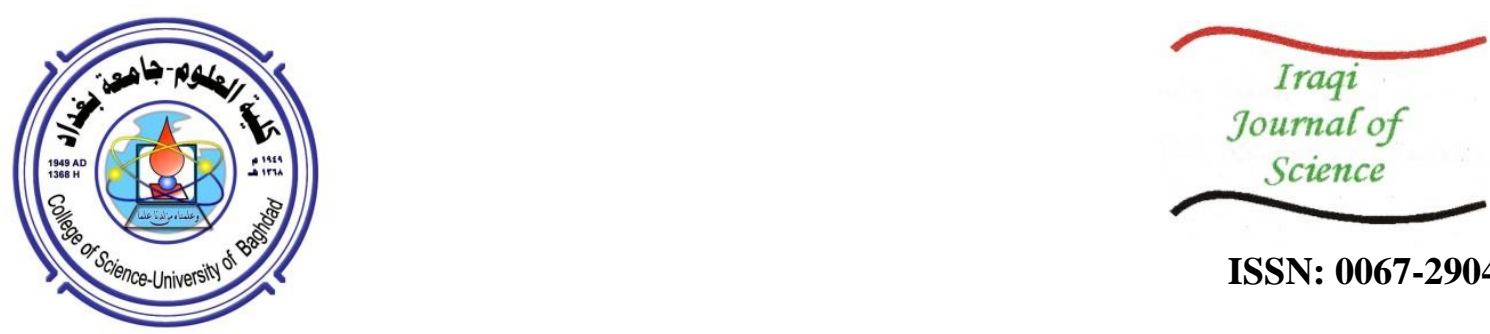

ISSN: 0067-2904

\title{
Some Properties of Fuzzy Anti-Inner Product Spaces
}

\author{
Radhi I. M. Ali, Esraa A. Hussein" \\ Department of Mathematics, College of Science for Women, University of Baghdad, Baghdad, Iraq \\ Received: 19/11/2019 \\ Accepted: 28/12/2019

\begin{abstract}
:
In this paper, the definition of fuzzy anti-inner product in a linear space is introduced. Some results of fuzzy anti-inner product spaces are given, such as the relation between fuzzy inner product space and fuzzy anti-inner product. The notion of minimizing vector is introduced in fuzzy anti-inner product settings.
\end{abstract}

Keywords: fuzzy anti-inner product, fuzzy anti-norm, fuzzy inner product.

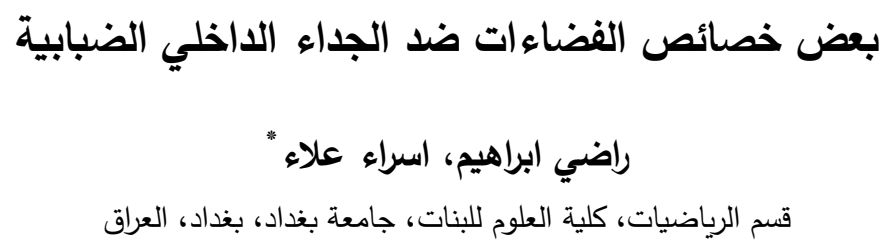

\section{Introduction}

Kohli and Kumar, in 1993 [1], introduced the definition of the fuzzy inner product space and fuzzy co-inner product space. In 1997, Alsina et al [2] introduced the ideal of probabilistic inner product space. After that, in 2010, Hasankhain et al. [3] introduced some properties of fuzzy Hilbert spaces and norm of operators. In 2013, the fuzzy real inner product space and its properties were proved by Mukherjee and Bag [4]. Finally, a note on fuzzy Hilbert spaces was introduced by Daraby et al. in 2016 [5].

\section{Preliminaries}

This section consists of some definitions and results that will be needed later in this paper.

\section{Definition (2.1) [6]}

Assume that $V$ is a linear space over the field $C$ of complex numbers. A mapping $M^{*}: V^{2} \times C \rightarrow I$ satisfies the following conditions for all $x, y, z$ in $V$ and $t, s$ in $C$ :

(FIP1) $M^{*}(x+y, z,|t|+|s|) \geq \min \left\{M^{*}(x, z,|t|), M^{*}(y, z,|s|)\right\}$

$(\mathrm{FIP} 2) M^{*}(x, y,|t s|) \geq \min \left\{M^{*}\left(x, x,|t|^{2}\right), M^{*}\left(y, y,|s|^{2}\right)\right\}$

(FIP3) $M^{*}(x, y, t)=M^{*}(y, x, \bar{t})$

(FIP4) $M^{*}(\alpha x, y, t)=M^{*}\left(x, y, \frac{t}{\alpha}\right), 0 \neq \alpha \in C$

(FIP5) for all $t \in C \backslash R^{+}, M^{*}(x, x, t)=0$

(FIP6) $\forall t>0, M^{*}(x, x, t)=1$ if and only if $x=\underline{0}$

(FIP7) $M^{*}(x, x,):. R \rightarrow I$ is a monotonic non-decreasing function of $R$ and $\lim _{t \rightarrow \infty} M^{*}(x, x, t)=1$, where $M^{*}$ is called a fuzzy inner product function on $V$ and $\left(V, M^{*}\right)$ is called a fuzzy inner product space.

\section{Definition (2.2) [7]}

Let $V$ be a linear space over a field $F$. A fuzzy set $\mathcal{N}: V \times R \rightarrow I$ such that the following holds for all $u, v$ in $V$ and $c$ in $F$ : 
$(\mathcal{N} 1)$ for all $t \in R$ with $t \leq 0, \mathcal{N}(u, t)=1$;

$(\mathcal{N} 2)$ for all $t \in R$ with $t>0, \mathcal{N}(u, t)=0$ if and only if $u=\underline{0}$;

$(\mathcal{N} 3)$ for all $t \in R$ with $t>0, \mathcal{N}(c u, t)=\mathcal{N}\left(u, \frac{t}{|c|}\right)$ if $0 \neq c \in F$

$(\mathcal{N} 4)$ for all $\mathrm{s}, \mathrm{t} \in R, \mathcal{N}(u+v, s+t) \leq \max \{\mathcal{N}(u, s), \mathcal{N}(v, t)\}$

$(\mathcal{N} 5) \mathcal{N}(u, t)$ is a decreasing function of $t \in R$ and $\operatorname{Lim}_{t \rightarrow \infty} \mathcal{N}(u, t)=0$,

where $\mathcal{N}$ is said to be a fuzzy anti-norm on $V$ and $(V, \mathcal{N})$ is called a fuzzy anti-normed linear space.

Later on, the following condition of fuzzy norm $\mathcal{N}$ will be required:

$(\mathcal{N} 6)$ for all $\mathrm{t} \in R$ with $t>0, \mathcal{N}(u, t)<1$ implies $u=\underline{0}$.

Definition (2.3) [7]

Le $\mathcal{N}$ be a fuzzy anti-norm on $V$ satisfying $(\mathcal{N} 6)$. Define

$\|u\|_{\alpha}^{*}=\inf \{t>0: \mathcal{N}(u, t)<\alpha, \alpha \in(0,1]\}$.

\section{Theorem (2.4) [7]}

Let $(V, \mathcal{N})$ be a fuzzy anti-normed linear space. Then $\left\{\|u\|_{\alpha}^{*}: \alpha \in(0,1]\right\}$ is a decreasing family of norms on $V$.

\section{Fuzzy anti-inner product space}

The definition of fuzzy anti-inner product space on a complex linear space is introduced and some of its results are investigated.

\section{Definition (3.1) [8]}

Assume that $V$ is a linear space over the filed $C$ of complex numbers. Define

$M^{\circ}: V^{2} \times C \rightarrow I$ to be a mapping such that the following holds for all $x, y$ in $V$ and $t, s$ in $C$ :

Fa-IP1 $) M^{\circ}(x+y, z,|t|+|s|) \leq \max \left\{M^{\circ}(x, z,|t|), M^{\circ}(y, z,|s|)\right\}($

Fa-IP2) $M^{\circ}(x, y,|t s|) \leq \max \left\{M^{\circ}\left(x, x,|t|^{2}\right), M^{\circ}\left(y, y,|s|^{2}\right)\right\}($

Fa-IP3 $M^{\circ}(x, y, t) \leq M^{\circ}(y, x, \bar{t})($

Fa-IP4) $M^{\circ}(\alpha x, y, t) \leq M^{\circ}\left(x, y, \frac{t}{|\alpha|}\right), 0 \neq \alpha \in C($

Fa-IP5) $M^{\circ}(x, x, t)=1 \quad \forall t \in C \backslash R^{+}($

Fa-IP6) $\forall t>0, M^{\circ}(x, x, t)=\underline{0}$ if and only if $x=\underline{0}$

(Fa-IP7) $M^{\circ}(x, x,):. R \rightarrow I$ is a monotonic non-increasing function of $R$

and $\lim _{t \rightarrow \infty} M^{\circ}(x, x, t)=0$.

Where $M^{\circ}$ is called a fuzzy anti-inner product function on $V$ and $\left(V, M^{\circ}\right)$ is called a fuzzy anti-inner product space.

Example (3.2)

Assume that $(V,<,>)$ is an inner product space over $C$. A function $M^{\circ}: V^{2} \times C \rightarrow I$ is defined by

$$
M^{\circ}(x, y, t)= \begin{cases}1 & \text { if } t \leq|<x, y>| \\ 0 & \text { if } t>|<x, y>| \\ 1 & \forall t \in C \backslash R\end{cases}
$$

Then $M^{\circ}$ is a fuzzy anti- inner product space on $V$.

\section{Proof:}

(Fa-IP1) Consider the following cases:

Case (i) if one of $|t| \leq|<x, z\rangle|| s,|\leq|<y, z\rangle \mid$ holds, then

$\max \left\{M^{\circ}(x, z,|t|), M^{\circ}(y, z,|s|)\right\}=1$ and obviously

$M^{\circ}(x+y, z,|t|+|s|)=0 \leq \max \left\{M^{\circ}(x, z,|t|), M^{\circ}(y, z,|s|)\right\}$

Case (ii) let $|t|>|\langle x, z\rangle|$ and $|s|>|<y, z\rangle \mid$

$\Rightarrow|t|+|s|>|<x+y, z>|$

$\therefore M^{\circ}(x+y, z,|t|+|s|)=0 \leq \max \left\{M^{\circ}(x, z,|t|), M^{\circ}(y, z,|s|)\right\}$

(Fa-IP2) We observe that $\left.|s|^{2}\right\rangle|<x, x\rangle \mid$ and $\left.|t|^{2}\right\rangle|<y, y>|$

$\Rightarrow|s|^{2} \cdot|t|^{2}>|<x, x>| \cdot|<y, y>|=\|x\|^{2} \cdot\|y\|^{2}$

$|s| .|t|>\|x\| \cdot\|y\| \quad \Rightarrow$

$|s t|>\|x\| \cdot\|y\| \quad \Rightarrow$

so (Fa-IP2) follows.

Next (Fa-IP3) (Fa-IP5) and (Fa-IP7) hold obviously.

(Fa-IP4) If $t \in\left(C \backslash R^{+}\right)$, then the result is obvious. 
For $t \in R^{+}, 0 \neq \alpha \in C$, then the property follows from the fact that

$|<\alpha x, y>|=|\alpha||<x, y>|$

(Fa-IP6) If $x=0 \Rightarrow\langle x, x>=0 \Rightarrow \forall t>0,|<x, x>|>t$

$$
\Rightarrow M^{\circ}(x, x, t)=0
$$

Conversely, if $\forall t>0, M^{\circ}(x, x, t)=0 \Rightarrow \forall t>0,|<x, x>|>t$

$$
\Rightarrow\langle x, x\rangle=0 \Rightarrow x=0 \text {. }
$$

This completes the proof.

\section{Proposition (3.3) [8]}

Let $\left(V, M^{\circ}\right)$ be a fuzzy anti-inner product space. Then for $x, y, z$ in $V$ and $s, t$ in $C$

(i) $M^{\circ}(x, y+z,|t|+|s|) \leq M^{\circ}(x, y,|t|) \vee M^{\circ}(x, z,|s|)$

(ii) For $\alpha \in C$ and $\alpha \neq 0, M^{\circ}(\alpha x, y, t)=M^{\circ}(x, \alpha y, t)$

(iii) $\forall t \in R$ and $t>0, M^{\circ}(\underline{0}, \underline{0}, t) \leq M^{\circ}(x, y, t)$

Note (3.4) [8]

Assume that $M^{\circ}$ satisfies the condition:

(Fa-IP8) $\forall t>0, M^{\circ}\left(x, x, t^{2}\right)<1 \Rightarrow x=\underline{0}$

Let $\left(V, M^{\circ}\right)$ be a fuzzy anti-inner product space, satisfying (Fa-IP8). Then $\forall \alpha \in(0,1)$,

$\|x\|_{\alpha}^{*}=\wedge\left\{t>0: M^{\circ}\left(x, x, t^{2}\right) \leq 1-\alpha\right\}$ is a crisp norm on $V$, called the

$\alpha$-anti norm on $V$ generated from $M^{\circ}$.

In the sequel we shall consider the following condition:

(Fa-IP9) $\forall x, y$ in $V$ and $p, q$ in $R$,

$M^{\circ}\left(x+y, x+y, 2 q^{2}\right) \vee M^{\circ}\left(x-y, x-y, 2 p^{2}\right) \leq M^{\circ}\left(x, x, p^{2}\right) \vee M^{\circ}\left(y, y, q^{2}\right)$

\section{Theorem (3.5) [8]}

Let $M^{\circ}$ be a fuzzy anti-inner product on the $V$ defined function $\mathcal{N}$, as follows:

$\mathcal{N}(x, t)=M^{\circ}\left(x, x, t^{2}\right) \quad \forall t \in R$ and $t>0$

$=1 \quad \forall t \in R$ and $t \leq 0$

Then $\mathcal{N}$ is a fuzzy anti-norm on $V$.

From now on, if (Fa-IP8) and (Fa-IP9) hold for each $\alpha \in(0,1)$, then

$\|x\|_{\alpha}^{*}=\Lambda\left\{t>0: M^{\circ}\left(x, x, t^{2}\right) \leq 1-\alpha\right\}$ is an ordinary anti-norm on $V$ satisfying parallelogram law.

So, by using polarization identity, one can get an ordinary inner product, called the $\alpha$-anti-inner product, as follows:

where $\quad X_{\alpha}^{*}=\frac{1}{4}\left(\|x+y\|_{\alpha}^{* 2}-\|x-y\|_{\alpha}^{* 2}\right)$

$$
<x, y>_{\alpha}^{*}=X_{\alpha}^{*}+i Y_{\alpha}^{*}
$$

and $\quad Y_{\alpha}^{*}=\frac{1}{4}\left(\|x+i y\|_{\alpha}^{* 2}-\|x-i y\|_{\alpha}^{* 2}\right)$, where $\alpha \in(0,1)$.

\section{Definition (3.6)}

$V$ is said to be anti-level complete (AL-complete). If $\left(V, M^{\circ}\right)$ is a fuzzy anti-inner product space satisfying (Fa-IP8) for any $\in(0,1)$, then every Cauchy sequence converges in $V$ w.r.t the $\alpha$-antinorm $\|x\|_{\alpha}^{*}$ generated by the fuzzy anti-norm $\mathcal{N}$ which is induced by fuzzy anti-inner product $M^{\circ}$.

\section{Theorem (3.7) (Minimizing vector)}

Let $\left(V, M^{\circ}\right)$ be a fuzzy anti-inner product space satisfying (Fa-IP8) and (Fa-IP9), let M $(\neq \varnothing)$ be the convex subset of $V$ which is anti-level complete, and let $x \in V$. Then for each $\alpha \in(0,1), \exists$ $y_{\alpha}^{*}$ in $\mathrm{M}$ such that

$$
\begin{array}{ll}
m_{y_{\alpha}^{*}}^{(\alpha)^{*}}=\inf _{y \in M}\left\{m_{y}^{(\alpha)^{*}}\right\} & , \text { where } \\
& m_{y}^{(\alpha)^{*}}=\Lambda\left\{t \in R^{+}, \mathcal{N}(x-y, t) \leq 1-\alpha\right\}
\end{array}
$$

$M^{\circ} . \quad \mathcal{N}$ is the fuzzy anti-norm induced by fuzzy anti-inner

\section{Proof:}

We note that if $\left(V, M^{\circ}\right)$ is a fuzzy anti-inner product space, then for each $\alpha \in(0,1),\left(V,\|\cdot\|_{\alpha}^{*}\right)$ is a crisp anti- normed linear space satisfying the parallelogram law. Again

$m_{y}^{(\alpha)^{*}}=\|x-y\|_{\alpha}^{*}$.

Hence the result follows from the corresponding crisp minimization vector theorem in $\left(V,\|.\|_{\alpha}^{*}\right)$. 


\section{Theorem (3.8)}

$M^{\circ}$ is a fuzzy anti-inner product space on $V$ if and only if $1-M^{\circ}$ is a fuzzy inner product space on $V$.

Proof: For $x, y, z$ in $V$ and $t, s$ in $C$

(Fa-IP1) $\max \left\{M^{\circ}(x, z,|t|), M^{\circ}(y, z,|s|)\right\}$

$=1-\max \left\{M^{\circ}(x, z,|t|), M^{\circ}(y, z,|s|)\right\}$

$=\min \left\{M^{*}(x, z,|t|), M^{*}(y, z,|s|)\right\}$

$\leq M^{*}(x+y, z,|t|+|s|)$

$$
\geq 1-M^{*}(x+y, z,|t|+|s|)=M^{\circ}(x+y, z,|t|+|s|)
$$

(Fa-IP2) $\max \left\{M^{\circ}\left(x, x,|s|^{2}\right), M^{\circ}\left(y, y,|t|^{2}\right)\right\}$

$=1-\max \left\{M^{\circ}\left(x, x,|s|^{2}\right), M^{\circ}\left(y, y,|t|^{2}\right)\right\}$

$=\min \left\{M^{*}\left(x, x,|s|^{2}\right), M^{*}\left(y, y,|t|^{2}\right)\right\}$

$\leq M^{*}(y, z,|s t|)$

$\geq 1-M^{*}(y, z,|s t|)=M^{\circ}(y, z,|s t|)$

(Fa-IP3) $M^{\circ}(x, y, t)=1-M^{*}(x, y, t)$

$=1-M^{*}(y, x, \bar{t})=M^{\circ}(y, x, \bar{t})$

(Fa-IP4) $M^{\circ}(\alpha x, y, t)=1-M^{*}(\alpha x, y, t) \quad$ for $0 \neq \alpha \in C$

$=1-M^{*}\left(x, y, \frac{t}{|\alpha|}\right)=M^{\circ}\left(x, y, \frac{t}{|\alpha|}\right)$

(Fa-IP5) $\quad M^{\circ}(x, x, t)=1-M^{*}(x, x, t)=1-0=1 \quad \forall t \in C \backslash R^{+}$

(Fa-IP6) $M^{\circ}(x, x, t)=1-M^{*}(x, x, t) \Leftrightarrow x=0$

$=1-1 \Leftrightarrow \quad x=0$

(Fa-IP7) We have $M^{*}(x, x,):. R \rightarrow I \quad$ is a monotonic non-decreasing function and $\lim _{t \rightarrow \infty} M^{*}(x, x, t)=1$

then $M^{\circ}(x, x,):. R \rightarrow I$ is a monotonic non-increasing function and

$$
\lim _{t \rightarrow \infty} M^{\circ}(x, x, t)=1-\lim M_{t \rightarrow \infty}^{*}(x, x, t)=1-1=0
$$

\section{Theorem (3.9)}

Hence $\left(V, M^{\circ}\right)$ is a fuzzy anti-inner product space.

Let $(V, \mathcal{N})$ be a fuzzy anti-normed linear space. Suppose that for $x, y, z$ in $V$ and $t, s, r$ in $C$,

$$
\max \{\mathcal{N}(x,|s t|), \mathcal{N}(y,|s t|)\} \leq \max \left\{\mathcal{N}\left(x,|s|^{2}\right), \mathcal{N}\left(y,|t|^{2}\right)\right\}
$$

Define $M^{\circ}: V^{2} \mathrm{x} C \rightarrow I$ as $M^{\circ}(x, y, s+t)=1$

if $x=y$ and $s+t \in C \backslash R^{+}$and elsewhere as

$$
M^{\circ}(x, y, s+t)=\mathcal{N}(x,|s|) \wedge \mathcal{N}(y,|t|)
$$

Then $M^{\circ}$ is a fuzzy anti-inner product on $V$.

Proof: For $x, y, z$ in $V$ and $t, s$ in $C$,

$$
\text { (Fa-IP1) } \begin{aligned}
M^{\circ}(x+y, z,|s|+|t|)= & M^{\circ}(x+y, z,|s|+|t|+0) \\
= & \mathcal{N}(x+y,|s|+|t|) \wedge \mathcal{N}(z, 0) \\
= & \mathcal{N}(x+y,|s|+|t|) \\
& \leq \max \{\mathcal{N}(x,|s|), \mathcal{N}(y,|t|)\} \\
= & \max \left\{M^{\circ}(x, z,|s|), M^{\circ}(y, z,|t|)\right\}
\end{aligned}
$$

Fa-IP2) $M^{\circ}(x, y,|s t|)=\mathcal{N}(x,|s t|) \wedge \mathcal{N}(y,|s t|)($

$=\max \{\mathcal{N}(x,|s t|), \mathcal{N}(y,|s t|)\}$

$\leq \max \left\{\mathcal{N}\left(x,|s|^{2}\right), \mathcal{N}\left(y,|t|^{2}\right)\right\}$

$=\max \left\{M^{\circ}\left(x, x,|s|^{2}\right), M^{\circ}\left(y, y,|t|^{2}\right)\right\}$

(Fa-IP3) $M^{\circ}(x, y, t)=\mathcal{N}(x,|t|)=\mathcal{N}(x, \overline{|t|})$

$=M^{\circ}(x, y, \bar{t})=\mathcal{N}(y, \overline{|t|})$

(Fa-IP4) For $\alpha \neq 0$

$$
=M^{\circ}(y, x, \bar{t})
$$

$$
M^{\circ}(\alpha x, y, t)=\mathcal{N}(\alpha x,|t|)=\mathcal{N}\left(x, \frac{|t|}{|\alpha|}\right)=M^{\circ}\left(x, y, \frac{|t|}{|\alpha|}\right)
$$


(Fa-IP5) By definition $\forall t \in C \backslash R^{+}, M^{\circ}(x, y, t)=1$

(Fa-IP6) $\quad M^{\circ}(x, x, t)=0 \quad \forall t>0$

$\Leftrightarrow \mathcal{N}(x,|t|)=0 \quad \forall t>0$

$\Leftrightarrow x=0$

Hence $\left(V, M^{\circ}\right)$ is a fuzzy anti-inner product space.

Theorem (3.10)

Let $\left(V, M^{\circ}\right)$ be a fuzzy anti-inner product space satisfying (Fa-IP8) and (Fa-IP9) and $\langle,\rangle_{\alpha}$ be $\alpha$ anti-inner product $\forall \alpha \in(0,1)$.

Define a function

$M^{\circ}: V^{2} \mathrm{x} C \rightarrow I$ as $M^{\circ}(x, y, s+t)=1$

if $x=y$ and $t \in C \backslash R^{+}$and elsewhere as

$$
M^{\circ}(x, y, t)=\wedge\left\{\alpha \in(0,1):\left|<,>_{\alpha}\right| \geq|t|\right\}
$$

Then $M^{\circ}$ is a fuzzy anti-inner product on $V$ if $\left|<,>_{\alpha}\right|$ is a decreasing function of $R$.

Proof: For $x, y, z$, in $V$ and $t, s$, in $C$,

(Fa-IP1) To prove that $M^{\circ}(x+y, z,|s|+|t|) \leq \max \left\{M^{\circ}(x, z,|s|), M^{\circ}(y, z,|t|)\right\}$

Let $p=M^{\circ}(x, z,|s|)$ and $q=M^{\circ}(y, z,|t|)$.

Without loss of generality, assume that $p \leq q$ and let $0<r<p \leq q$

Then $\exists 0<\alpha<r$ such that $|<x, z\rangle_{\alpha}|>| s \mid$ and

$\exists 0<\beta<r$ such that $\left|<y, z>_{\beta}\right|>|t|$

Let $0<\gamma=\alpha \vee \beta<r$. Thus

and

$$
\left|<x, z>_{\gamma}\right|>\left|<x, z>_{\alpha}\right|>|s|
$$

[ Since $\left|<,>_{\alpha}\right|$ is a decreasing function ]

$$
\left|<y, z>_{\gamma}\right|>\left|<y, z>_{\beta}\right|>|t|
$$

Now $\left|<x+y, z>_{\gamma}\right|=\left|<x, z>_{\gamma}+<y, z>_{\gamma}\right|$

$\leq\left|<x, z>_{\gamma}\right|+\left|<y, z>_{\gamma}\right|$

$>|s|+|t|$

Therefore $M^{\circ}(x+y, z,|s|+|t|) \leq \gamma<r$, since $r>0$, thus

(Fa-IP2) To prove that

$$
M^{\circ}(x+y, z,|s|+|t|) \leq \max \left\{M^{\circ}(x, z,|s|), M^{\circ}(y, z,|t|)\right\}
$$

$M^{\circ}(x, y,|s t|) \leq \max \left\{M^{\circ}\left(x, y,|s|^{2}\right), M^{\circ}\left(x, y,|t|^{2}\right)\right\}$

Let $p=M^{\circ}\left(x, y,|s|^{2}\right)$ and $q=M^{\circ}\left(x, y,|t|^{2}\right)$.

Without loss of generality, assume that $p \leq q$ and let $0<r<p \leq q$

Then $\exists 0<\alpha<r$ such that $\left|<x, y>_{\alpha}\right|>|s|^{2}$ and

$\exists 0<\beta<r$ such that $\left|<x, y>_{\beta}\right|>|t|^{2}$

Let $0<\gamma=\alpha \vee \beta<r$. Thus

and

$$
\left|<x, y>_{\gamma}\right|>\left|<x, y>_{\alpha}\right|>|s|^{2}
$$

[ Since $|<,\rangle_{\alpha} \mid$ is decreasing function ]

$$
\left|<x, y>_{\gamma}\right|>\left|<x, y>_{\beta}\right|>|t|^{2}
$$

Therefor $\left|<x, y>_{\gamma}\right|^{2}>|s|^{2} .|t|^{2} \Rightarrow\left|<x, y>_{\gamma}\right|>|s t|$

therefore $M^{\circ}(x, y,|s t|) \leq \gamma<r$, since $r>0$ is arbitrary, thus

$$
M^{\circ}(x, y,|s t|) \leq \max \left\{M^{\circ}\left(x, y,|s|^{2}\right), M^{\circ}\left(x, y,|t|^{2}\right)\right\}
$$

(Fa-IP3) For $t \in C, M^{\circ}(x, y, t)=M^{\circ}(x, y, \bar{t})=1$

if $x=y$ and $\forall t \in C \backslash R^{+}$

Now let $t \in C$ and $x \neq y$, then

(Fa-IP4) For $c \in C$,

$$
\begin{gathered}
M^{\circ}(x, y, t)=\Lambda\left\{\alpha \in(0,1):\left|<x, y>_{\alpha}\right| \geq|t|\right\} \\
=\Lambda\left\{\alpha \in(0,1):\left|<x, y>_{\alpha}\right| \geq|\bar{t}|\right\} \\
=M^{\circ}(x, y, \bar{t})
\end{gathered}
$$$$
M^{\circ}(c x, y, t)=\Lambda\left\{\alpha \in(0,1):\left|<c x, y>_{\alpha}\right| \geq|t|\right\}
$$ 
(Fa-IP5) By definition $M^{\circ}(x, x, t)=1 \quad \forall t \in C \backslash R^{+}$

$$
\begin{gathered}
=\wedge\left\{\alpha \in(0,1):|c|\left|<x, y>_{\alpha}\right| \geq|t|\right\} \\
=\wedge\left\{\alpha \in(0,1):|c|\left|<x, y>_{\alpha}\right| \geq \frac{|t|}{|c|}\right\} \\
=M^{\circ}\left(x, y, \frac{t}{|c|}\right)
\end{gathered}
$$

(Fa-IP6) $\forall t>0, M^{\circ}(x, x, t)=0$

$$
\Leftrightarrow \wedge\left\{\alpha \in(0,1):|<x, x\rangle_{\alpha}|\geq| t \mid\right\}=0
$$

$\Leftrightarrow<x, x\rangle_{\alpha}=0$

$$
\Leftrightarrow x=\underline{0}
$$

$\left(\right.$ Fa-IP7) $\forall t>0, M^{\circ}(x, x, t)=\Lambda\left\{\alpha \in(0,1):\left|<x, x>_{\alpha}\right| \geq|t|\right\}$

$$
\begin{aligned}
& =\wedge\left\{\alpha \in(0,1):\|x\|_{\alpha}{ }^{2} \geq|t|\right\} \\
& =\wedge\left\{\alpha \in(0,1):\|x\|_{\alpha} \geq \sqrt{t}\right\}
\end{aligned}
$$

Now $t_{1}<t_{2} \Rightarrow \sqrt{t_{1}}<\sqrt{t_{2}}$

$$
\begin{gathered}
\Rightarrow\left\{\alpha \in(0,1):\|x\|_{\alpha} \geq \sqrt{t_{1}}\right\} \subset\left\{\alpha \in(0,1):\|x\|_{\alpha} \geq \sqrt{t_{2}}\right\} \\
\Rightarrow \wedge\left\{\alpha \in(0,1):\|x\|_{\alpha} \geq \sqrt{t_{1}}\right\} \leq \Lambda\left\{\alpha \in(0,1):\|x\|_{\alpha} \geq \sqrt{t_{2}}\right\}
\end{gathered}
$$

$\Rightarrow M^{\circ}\left(x, x, t_{1}\right) \leq M^{\circ}\left(x, x, t_{2}\right)$

Therefore $M^{\circ}(x, x,):. R^{+} \rightarrow I$ is decreasing and $\lim _{t \rightarrow \infty} M^{\circ}(x, x, t)=0$.

Thus $M^{\circ}$ is a fuzzy anti-inner product on $V$.

\section{References}

1. Kohli, J.K. and Rajesh K. 1993. On fuzzy inner product spaces \& fuzzy co inner product spaces, FSS, 53(1993): 227-232.

2. Alsina, C., Schweizer, B., Sempi, C. and Sklar, A. 1997. On the definition of a probabilistic inner product space, Rendiconti di Matematica, Serie VII, 17(1997): 115-127.

3. Hasankhani, A., Nazari, A. and Saheli, M. 2010. Some properties of fuzzy Hilbert spaces and norm of operators, Iranian Journal of Fuzzy Systems, 7(3): 129-157.

4. Mukherjee, S. and Bag, T. 2013. Fuzzy real inner product space and its properties, Annals of Fuzzy Mathematics and Informatics, 6(2): 377-389.

5. Daraby, B., Solimani, Z. and Rahimi, A. 2016. A note on fuzzy Hilbert spaces, Journal of Intelligent and Fuzzy Systems, 31: 313-319

6. Majumdar, P. and Samanta, S.K. 2008. On Fuzzy Inner Product Spaces, The J.Fuzzy Math., 16 (2): 377-392.

7. Iqbal Jebril, H. and Samanta, T.K. 2010. Fuzzy Anti-Normed space, Journal of Mathematics Technology, February, 66-77.

8. Parijat S. 2019. Fuzzy Anti Inner Product Space, International Journal of Applied Engineering Research, 14(19): 3756-3762. 